had been felt from the exterior was a tumour about the size of a goose's egg, growing inwards from the anterior end of the bladder. The posterior half of the organ appeared quite normal, and in order to obtain a better view of the tumour the bladder was cut across just in front of its neck, and then everted. The accompanying photograph shows the appearance which the parts then presented. The surface of the tumour was rough, ulcerating, and at some places covered with small blood coagula. Its consistence was rather firmer than that of normal liver, and on section it presented a white surface finely mottled with grey.

Around the base of the tumour the mucous membrane of the bladder showed a fringe of growths which in size and shape resembled the large papilla of the rumen (see Fig.), but elsewhere the lining membrane of the bladder was normal. The urine contained in the organ was turbid and blood-tinged, but it had no odour of putridity.

The abdominal and thoracic viscera were found to be normal, and there was no evidence of metastasis in the pelvic or other lymphatic glands.

Subsequent microscopic examination showed that the tumour had the histology of a carcinoma (see Fig 2, Plate I.). The epithelial cells, like those of the normal bladder, are multiform in shape, but the majority are of the squamous type, and some of them are of very large size. Towards the centre of a few of the larger groups some of the cells have undergone colloid degeneration. The stroma of the tumour is sparing in amount, and for the most part spindle-celled. Clusters of leucocytes are present at places, both in the epithelium and the stroma.

If, as the history indicated, the tumour had been in existence for about a year, it is somewhat remarkable that secondary tumours had not formed even in the adjacent lymphatic glands.

\title{
GENERALISED CARCINOMA IN A HORSE" NEGATIVE RESULT OF AN ATTEMPT TO TRANSMIT THE DISEASE BY INOCULATION.
}

By the Same.

IN the month of December last I had submitted to me by my colleague Professor Penberthy a small piece of a tumour from the tail of a horse for microscopic examination. The examination showed that the growth was a carcinoma, and a few days later Professor Penberthy amputated the tail above the apparent upper limit of the tumour, which, unfortunately, was almost close to the root. The situation and extent of the growth are shown in Fig. I, which is a photograph of the amputated portion of the tail, viewed from its under aspect after removal of the hair.

The patient, a bay gelding over twenty years of age, remained in the College Infirmary till the 23 rd January last, when it fell down in its box and died in less than half an hour. Just before death it was observed that the visible mucous membranes had become very pale. 
The wound of the stump had not cicatrised, and the animal's appetite had failed. For some days before death the respirations had been hurried. The following are the notes of the post-mortem examination, which was begun one hour after death :-

Autopsy. - Carcase poor but not emaciated. The surface of the stump of the tail, which is about four inches in length, has a raw, ulcerating appearance. On the under aspect of the stump, and extending forwards above the rectum, there is an irregular mass of tumour growth. On both sides the superficial inguinal glands are enlarged, and apparently invaded; the left group, which is the larger of the two, has the volume of a hen's egg.

The peritoneal cavity contains a large quantity (estimated at a gallon) of liquid blood. The stomach and intestines are normal as regards their exterior. Three of the mesenteric glands are enlarged

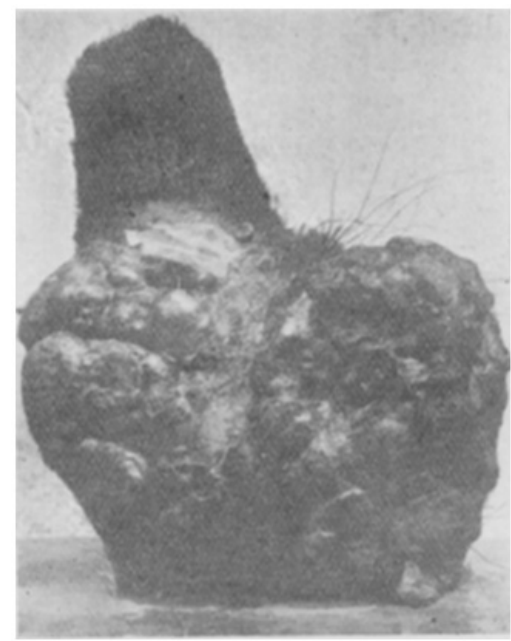

FIG. 1.

Primary carcinoma of the tail.

and:apparently invaded by new growth, the largest one being about the size of a pullet's egg. Several of the glands of the large colon are similarly enlarged and invaded.

The spleen weighs $13^{3}$. Ibs, and it contains a great number of tumours, varying in size from a hen's egg downwards. Some of the splenic lymphatic glands are also enlarged and invaded.

The liver is enormously enlarged, and weighs $46 \mathrm{lbs}$; it is crammed with tumours, the largest of which is about the size of a cocoa-nut. The intervening liver tissue is pale and easily lacerated.

The peritoneum on the right lobe is lacerated, and the liver substance is here partly infiltrated with blood. This appears to have been the source of the blood found in the peritoneal cavity.

Both kidneys are considerably enlarged, and together they weigh 6 Ibs. Both contain a considerable number of tumours, the largest of which are about walnut-size and the smallest like pins' heads. These 
tumours are all situated in the superficial part of the cortex, and some of them project freely above the kidney surface. The suprarenal bodies are normal.

In the substance of the pancreas there are several tumours, the largest being about half the size of a walnut.

The renal, lumbar, iliac, and pelvic lymphatic glands are more or less enlarged and invaded by new growth.

The terminal part of the posterior aorta contains an old thrombus, about 3 inches in length. The wall of the artery is here partially calcified, and the deeper strata of the thrombus are of a dirty white colour.

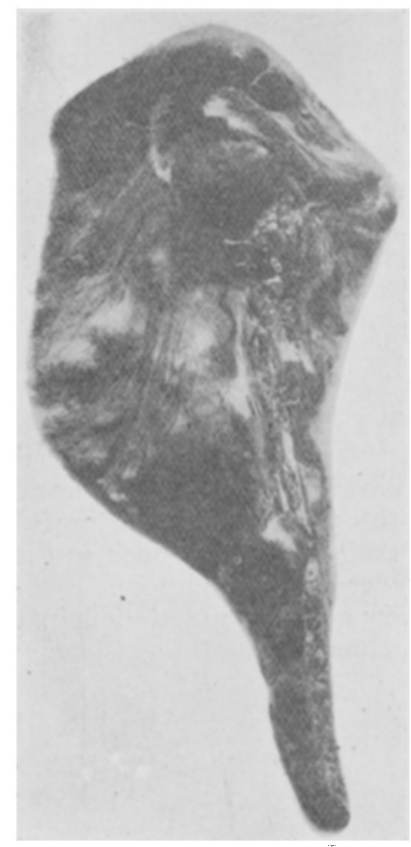

Fig. 2 ,

Spleen with secondary tumours.

In the muscular substance of the diaphragm there are about half a dozen small tumours, the largest not exceeding a hazel nut in size. Two larger tumours, one about the size of a pullet's egg and the other somewhat larger than a walnut, project freely from the abdominal surface of the diaphragm ; the smaller of the two is distinctly mushroom-shaped.

The pleural cavities contain a small amount of greyish turbid liquid. Projecting from one of the intercostal spaces on the left side there is a small mushroom-shaped tumour; another small growth is present in the intercostal space but under the pleura, which it only slightly projects.

Both lungs are crammed with tumours, from pin-head size up to the 
size of an orange. The distribution of the tumours is far from uniform, but it is impossible to find a cubic inch that is free from them.

The bronchial glands on both sides are three or four times the normal size, and are obviously invaded, but the tracheal group appears normal. The heart is normal.

The axillary lymphatic glands on both sides are enlarged and

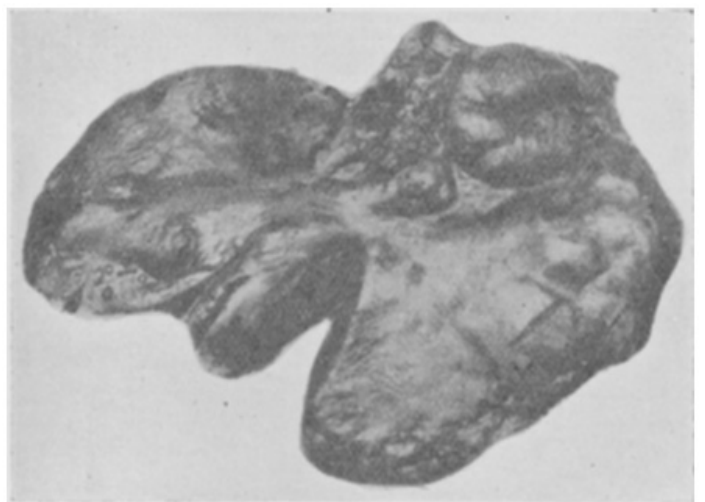

FIG. 3,

Liver with secondary tumours.

invaded, but the following groups are normal: popliteal, deep inguinal, precrural, prescapular, and pharyngeal.

Under the skin on both sides of the neck, in the fatty tissue beneath the mane, there are several small tumours.

The whole of the new growths mentioned in the above notes had similar macroscopic characters. In consistence they were a little

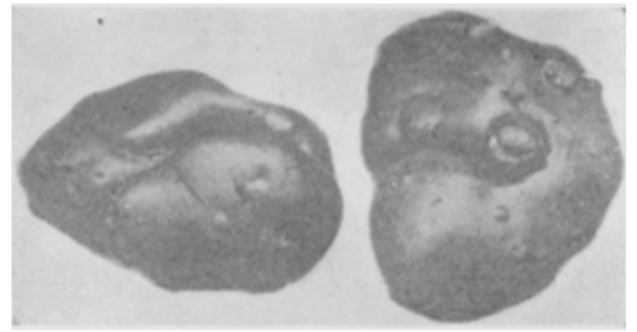

FIG. 4.

Kidneys with secondary turnours.

firmer than a normal lymphatic gland. In colour they were lightish grey, mottled with opaque white in the case of some parts of the larger tumours. The tissue of the tumours was easily lacerated or scraped down with a knife.

Microscopic examination of sections made from preserved portions of the primary growth in the tail (Fig. I, Plate I.), and of the 
secondary tumours in the spleen, liver, and lungs showed that all were carcinomata. In all of them the epithelial cells are of the small or glandular type, which suggests that the primary tumour may have had its starting point in connection with the sudoriparous glands of the tail. The amount of stroma is variable, but for the most part sparing.

Experiment.-The case appeared to present an eminently favourable opportunity for putting the parasitic theory of the cause of cancer to the test of experiment, and a pony was therefore inoculated in the following manner with material taken from the new growths.

On the 24th January (the day after the horse's death) a number of tumours in the spleen, liver, and lungs were first seared on the outside with a hot iron, and then incised with a sterile knife. The freshly exposed surfaces were scraped with the knife, and the material thus obtained was triturated with a quantity of sterile water. After the coarser particles had settled to the bottom, the opaque milky-looking liquid was used for inoculation, 2 cc. being injected subcutaneously on each side of the neck: and $5 \mathrm{cc}$. into the left jugular vein of an aged pony.

There was no apparent reaction at the points of inoculation in the neck, and the pony remained well and continued to improve in condition. It was killed on the 24 th June (five months after inoculation), and the post-mortem examination revealed nowhere any lesion that could be attributed to the inoculation. There was nothing to indicate the points of inoculation in the neck, and the adjacent lymphatic glands (prescapular), as well as all the other groups in the body, appcared quite normal. A number of calcified nodules, about the size of barley grains, were present in the left lung and in the liver, but with this exception the internal organs were normal.

It need hardly be said that the negative result of this experiment is not put forward as proof that the carcinomata are not the result of micro-parasitic irritation of the tissues, but at the same time it may reasonably be held that it is an item of evidence pointing in that direction, or at least indicating that in this particular case the carcinomatous disease was not of a parasitic nature. It may indeed be contended that a greater weight attaches to this experiment than to many others which resembled it in having negative results. In the great majority of the attempts that have been made to transmit carcinomatous disease by inoculation the experimental animal has not been of the same species as the one in which the tumour used to furnish the material for inoculation naturally arose. All experimentation with regard to the etiology of human carcinoma is thus open to the objection that the animal selected for experiment may be highly immune against the hypothetical germ of the human disease, and the objection is not disposed of by selecting the experimental animal from a species in which cases of carcinoma naturally occur ; for if any of the carcinomata are parasitic in their origin it is conceivable that, in spite of their similar histology, the carcinomata of different species of animals may be caused by distinct parasites.

To the experiment here recorded, however, no such objection can be taken, as the subject of the natural disease was a horse, and the attempt to infect was made on an animal of the same species and approximately of the same age. 
It may also be observed that the natural case which furnished the material for inoculation had a character favourable for the experimental transmission of the discase, assuming it to be caused by a parasite, viz., that of extreme malignancy.

Finally, it may be mentioned that in a former experiment, almost exactly parallel to the one here described, a like negative result was obtained (see this Journal, Vol. XIII. p. 342).

\section{A PECULIAR CASE OF INTUSSUSCEPTION OF THE SMALL INTESTINE OF THE HORSE.}

By the Same.

THE following case appears to merit description on account of its rarity. The subject was a chestnut mare, aged seven years, which died in the College Infirmary on the morning of the 9 th April last. The animal had been admitted on the previous day, on account of symptoms of severe abdominal pain. The post-mortem examination was made two hours after death, and the abdomen was then slightly

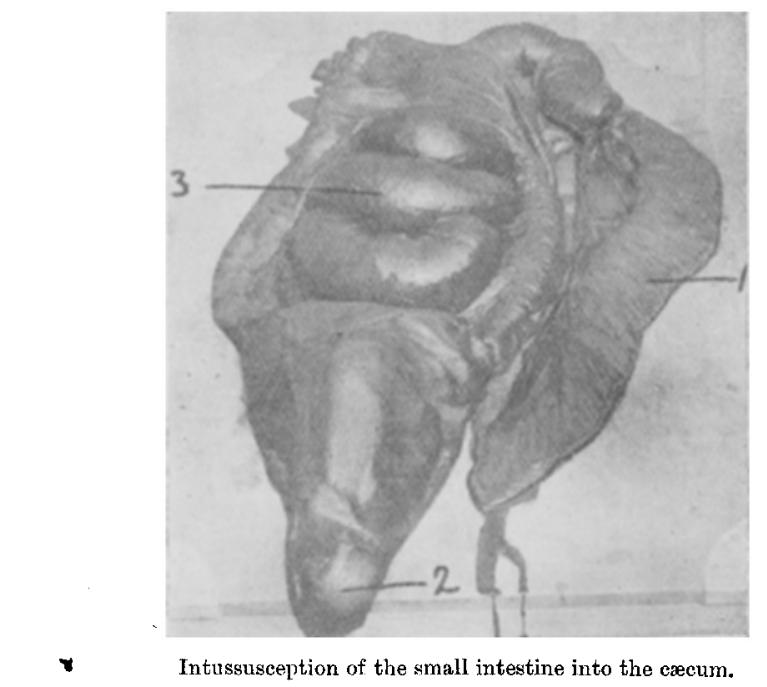

1, Ileum ; 2, point of cæeum ; 3, loops of intussuscepted ileum exposed by removal of part of excum.

tympanitic. When the abdominal wall was reflected the peritoneal cavity was found to contain a considerable quantity of deeply bloodtinged liquid, and displacement of the intestines showed that the mesenteric veins belonging to the posterior part of the small bowel were much distended with blood and had hamorrhages along their course. Further examination revealed the presence of an intussusception which appeared to begin about six inches from the termination of the ileum. As soon as these points had been determined the 